\title{
The Erdős-Sós Conjecture for Geometric Graphs $\|^{\dagger}$
}

\section{Luis Barba $^{1,2}$ Ruy Fabila-Monroy ${ }^{3} \mid$ Dolores Lara $^{4 \dagger}$ Jesús Leaños $^{5}$ Cynthia Rodríguez $^{6,7}$ Gelasio Salazar非 Francisco J. Zaragoza ${ }^{6}$}

\author{
${ }^{1}$ Boursier FRIA du FNRS, Département d'Informatique, ULB, Belgium \\ ${ }^{2}$ School of Computer Science, Carleton University, Canada \\ ${ }^{3}$ Departamento de Matemáticas, CINVESTAV, Mexico \\ ${ }^{4}$ Departament de Matemàtica Aplicada II, Universitat Politènica de Catalunya, Barcelona, Spain. \\ ${ }^{5}$ Unidad Académica de Matemáticas, UAZ. Mexico \\ ${ }^{6}$ Departamento de Sistemas, UAM Azcapotzalco, Mexico \\ ${ }^{7}$ Department of Combinatorics and Optimization, University of Waterloo, Canada \\ ${ }^{8}$ Instituto de Física, UASLP, Mexico
}

received $5^{\text {th }}$ July 2012, revised $19^{\text {th }}$ January 2013, accepted $8^{\text {th }}$ February 2013.

Let $f(n, k)$ be the minimum number of edges that must be removed from some complete geometric graph $G$ on $n$ points, so that there exists a tree on $k$ vertices that is no longer a planar subgraph of $G$. In this paper we show that $\left(\frac{1}{2}\right) \frac{n^{2}}{k-1}-\frac{n}{2} \leq f(n, k) \leq 2 \frac{n(n-2)}{k-2}$. For the case when $k=n$, we show that $2 \leq f(n, n) \leq 3$. For the case when $k=n$ and $G$ is a geometric graph on a set of points in convex position, we completely solve the problem and prove that at least three edges must be removed.

Keywords: extremal graph theory, geometric graph, spanning tree

\section{Introduction}

One of the most notorious problems in extremal graph theory is the Erdôs-Sós Conjecture, which states that every simple graph with average degree greater than $k-2$ contains every tree on $k$ vertices as a subgraph. This conjecture was recently proved true for all sufficiently large $k$ (unpublished work of Ajtai, Komlós, Simonovits, and Szemerédi).

In this paper we investigate a variation of this conjecture in the setting of geometric graphs. Recall that a geometric graph $G$ consists of a set $S$ of points in the plane (these are the vertices of $G$ ), plus a set of straight line segments, each of which joins two points in $S$ (these are the edges of $G$ ). In particular, any set $S$ of points in the plane in general position (no three of its points are collinear) naturally induces a complete geometric graph. For brevity, we often refer to the edges of this graph simply as edges of $S$. If

\footnotetext{
${ }^{\dagger}$ A previous version of this paper appeared in the conference proceedings of EUROCG’'12 [2].

¥Partially supported by CONACYT of Mexico, Grant 153984.

§Partially supported by CONACYT of Mexico, Grant 106432.
} 
$S$ is in convex position then $G$ is a convex geometric graph. A geometric graph is planar if no two of its edges cross each other. An embedding of an abstract graph $H$ into a geometric graph $G$ is an isomorphism from $H$ to a planar geometric subgraph of $G$. For $r \geq 0$, an $r$-edge is an edge of $G$ such that in one of the two open semi-planes defined by the line containing it, there are exactly $r$ points of $G$. The convex hull of $S$ is the intersection of all convex sets containing $S$. We will frequently need to refer to the vertices and edges at the boundary of the convex hull of $S$, which for brevity we will denote simply as convex hull vertices and convex hull edges of $S$.

In this paper all point sets are in general position and $G$ is a complete geometric graph on $n$ points. It is well known that for every integer $1 \leq k \leq n, G$ contains every tree on $k$ vertices as a planar subgraph [3]. Even more, it is possible to embed any such tree into $G$, when the image of a given vertex is prespecified [5].

Let $F$ be a subset of edges of $G$, which we call forbidden edges. If $T$ is a tree for which every embedding into $G$ uses an edge of $F$, then we say that $F$ forbids $T$. In this paper we study the question of what is the minimum size of $F$ so that there is a tree on $k$ vertices that is forbidden by $F$. Let $f(n, k)$ be the minimum of this number taken over all complete geometric graphs on $n$ points. As $f(2,2)=1$, $f(3,3)=2, f(4,3)=3, f(4,4)=2$ and $f(n, 2)=\left(\begin{array}{l}n \\ 2\end{array}\right)$, we assume through out the paper that $n \geq 5$ and $k \geq 3$.

We show the following bounds on $f(n, k)$.

\section{Theorem 1.1}

$$
\left(\frac{1}{2}\right) \frac{n^{2}}{k-1}-\frac{n}{2} \leq f(n, k) \leq 2 \frac{n(n-2)}{k-2}
$$

$$
2 \leq f(n, n) \leq 3
$$

In the case when $G$ is a convex complete geometric graph, we show that the minimum number of edges needed to forbid a tree on $n$ vertices is three.

An equivalent formulation of the problem studied in this paper is to ask how many edges must be removed from $G$ so that it no longer contains every planar subtree on $k$ vertices. A related problem is to ask how many edges must be removed from $G$ so that it no longer contains any planar subtree on $k$ vertices. For the case of $k=n$, in [6], it is proved that if any $n-2$ edges are removed from $G$, it still contains a planar spanning subtree. Note that if the $n-1$ edges incident to any vertex of $G$ are removed, then $G$ no longer contains a spanning subtree. In general, for $2 \leq k \leq n-1$, in [1], it is proved that if any set of $\left[\frac{n(n-k+1)}{2}\right\rceil-1$ edges are removed from $G$, it still contains a planar subtree on $k$ vertices. In the same paper it is also shown that this bound is tight—a geometric graph on $n$ vertices and a subset of $\left[\frac{n(n-k+1)}{2}\right\rceil$ of its edges are shown, so that when these edges are removed, every planar subtree has at most $k-1$ vertices. In [4] the authors study the seemingly unrelated (non-geometric) problem of packing two trees into planar ${ }^{(i)}$ (abstract) graphs. That is, given two trees on $n$ vertices, the authors consider the question of when it is possible to find a planar graph having both of them as spanning trees and in which the trees are edge disjoint. However, although theirs is a combinatorial question rather than

(i) A planar (abstract) graph is an abstract graph that can be embedded in the plane; the embedding may not be unique. 

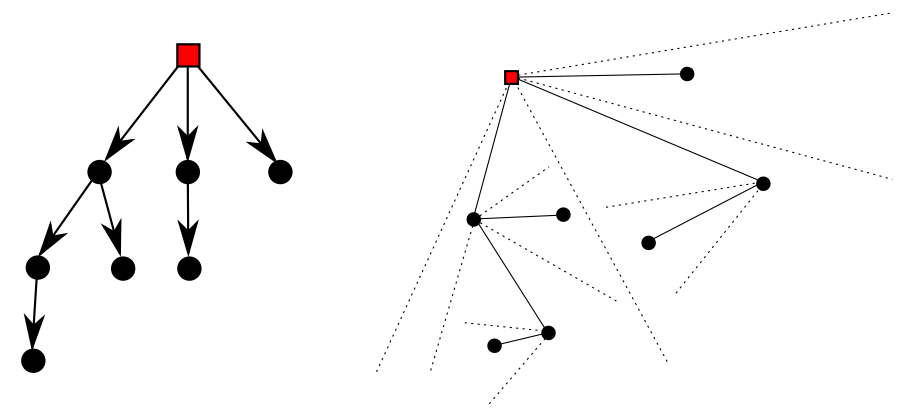

Fig. 1: An embedding of a tree using the algorithm.

geometric, their Theorem 2.1 implies our Lemma2.2. We provide a self contained proof of Lemma 2.2 for completeness.

A previous version of this paper appeared in the conference proceedings of EUROCG'12 [2].

\section{Spanning Trees}

In this section we consider the case when $k=n$. Let $T$ be a tree on $n$ vertices. Consider the following algorithm to embed $T$ into $G$. Choose a vertex $v$ of $T$ and root $T$ at $v$. For every vertex of $T$ choose an arbitrary order of its children. Suppose that the neighbors of $v$ are $u_{1}, \ldots, u_{m}$, and let $n_{1}, \ldots, n_{m}$ be the number of nodes in their corresponding subtrees. Choose a convex hull point $p$ of $G$ and embed $v$ into $p$. Sort the remaining points of $G$ counter-clockwise by angle around $p$. A wedge is a region of the plane bounded by two infinite rays sharing a common apex. Choose $m+1$ rays centered at $p$ so that the wedge between two consecutive rays is convex and between the $i$-th ray and the $(i+1)$-th ray there are exactly $n_{i}$ points of $G$. Let $S_{i}$ be this set of points. A convex hull vertex $q$ of $S_{i}$ is visible from $p$ if the line segment with endpoints $p$ and $q$ intersects the convex hull of $S$ only at $q$. For each $u_{i}$ choose a convex hull vertex of $S_{i}$ visible from $p$ and embed $u_{i}$ into this point. Recursively embed the subtrees rooted at each $u_{i}$ into $S_{i}$. Note that this algorithm provides an embedding of $T$ into $G$. We will use this embedding frequently throughout the paper. See Figure 1.

For every integer $n \geq 2$ we define a tree $T_{n}$ as follows: If $n=2$, then $T_{n}$ consists of only one edge; if $n$ is odd, then $T_{n}$ is constructed by subdividing once every edge of a star on $\frac{n+1}{2}$ vertices; if $n$ is even and greater than 2 , then $T_{n}$ is constructed by subdividing an edge of $T_{n-1}$. See Figure 2

We prove the lower bound of $f(n, n) \geq 2$ of Theorem 1.2 .

Theorem 2.1 If $G$ has only one forbidden edge, then any tree on $n$ vertices can be embedded into $G$, without using the forbidden edge.

Proof: Let $e$ be the forbidden edge of $G$. Let $T$ be a tree on $n$ vertices. Choose a root for $T$. Sort the children of each node of $T$, by increasing size of their corresponding subtrees. Embed $T$ into $G$ with the embedding algorithm, choosing at all times the rightmost point (the first point when sorting clockwise around the root) as the root of the next subtree. Suppose that $e$ is used in this embedding. Let $e:=p q$ so that $u$ is embedded into $p$ and $v$ is embedded into $q$ (note that $u$ is the parent of $v$ in $T$ ). 

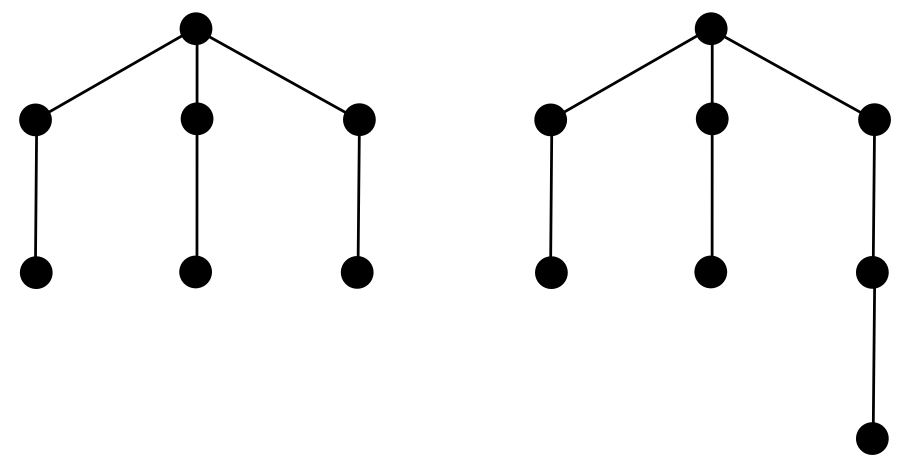

Fig. 2: $T_{7}$ and $T_{8}$.

Suppose that the subtree rooted at $v$ has $m \geq 2$ nodes. In the algorithm, we embedded this subtree into a set of exactly $m$ points. We chose a convex hull point $(q)$, of this set visible from $p$ to embed $v$. In this case we may choose another convex hull point visible from $p$ to embed $v$ and continue with the algorithm. Note that $p q$ is no longer used in the final embedding.

Suppose that $v$ is a leaf, and that $v$ has a sibling $v^{\prime}$ whose subtree has at least two nodes. Then we may interchange $v$ and $v^{\prime}$ in the order of the children of $u$, so that $e$ is no longer used in the embedding, or if it is, then $v^{\prime}$ is embedded into $q$, but then we proceed as above.

Suppose that $v$ is a leaf, has at least one sibling and all its siblings are leaves. The subtree rooted at $u$ is a star. We choose a point distinct from $p$ and $q$ in the point set where this subtree is embedded, and embed $u$ into this point. Afterward we join it to the remaining points. This produces an embedding that avoids $e$.

Assume then, that $v$ is a leaf and that it has no siblings. We distinguish the following cases:

1. $u$ has no siblings. In this case, the subtree rooted at the parent of $u$ is a path of length two. If $u$ has no grandparent then $n=3$ and $T$ can be trivially embedded into $G$ without using $e$. Suppose $u$ has a grandparent. In this case there are only four vertices to consider: $v, u$, the parent of $u$ and the grandparent of $u$. We keep the current location of the grandparent of $u$, and change the points into which the remaining vertices are embedded. This can always be done so that $e$ is not used in the embedding. All possible cases are shown in Figure 3

2. $u$ has a sibling $u^{\prime}$ whose subtree is not an edge. We may change the order of the siblings of $u$, with respect to their parent, so that the subtree rooted at $u^{\prime}$ will be embedded into the point set containing $p$ and $q$. In the initial order-increasing by size of their corresponding subtrees- $u^{\prime}$ is after $u$. We may assume that in the new ordering, the order of the siblings of $u$ before it, stays the same. Therefore $p$ is the rightmost point of the set into which the subtree rooted at $u^{\prime}$ will be embedded. Embed $u^{\prime}$ into $p$. Either we find an embedding not using $e$, or this embedding falls into one of the cases considered before.

\section{3. $u$ has at least one sibling, and the subtree at every sibling of $u$ is an edge.}

Suppose that $u$ has no grandparent; then $T$ is equal to $T_{n}$ and $n$ is odd. Let $w$ be the parent of $u$. Embed $w$ into $p$. Let $p_{1}, \ldots, p_{n-1}$ be the points of $G$ different from $p$ sorted counter-clockwise 

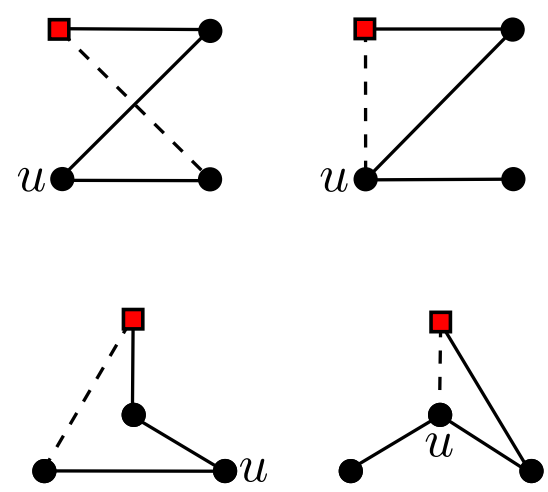
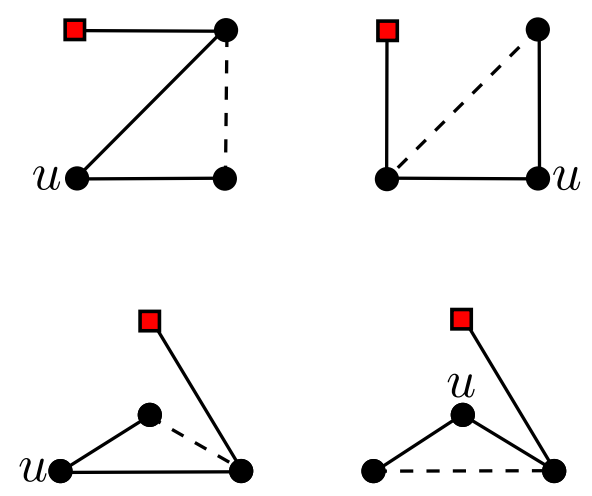

Fig. 3: The embedding of a path of length three. The grandparent of $u$ is highlighted and the forbidden edge is dashed.

by angle around $p$; choose $p_{1}$ so that the angle between two consecutive points is less than $\pi$. Let $u_{1}, \ldots, u_{(n-1) / 2}$ be the neighbors of $w$. Embed each $u_{i}$ into $p_{2 i-1}$ and its child into $p_{2 i}$. If $q$ equals $p_{2 j-1}$ for some $j$ then embed $u_{j}$ into $p_{2 j}$ and its child into $p_{2 j-1}$. This embedding avoids $e$.

Suppose that $w$ is the grandparent of $u$ and let $p^{\prime}$ be the point into which $w$ is embedded. Let $S$ be the point set into which the subtree rooted at the parent of $u$ is embedded. Note that $S$ has an odd number of points. We replace the embedding as follows. Sort $S$ counter-clockwise by angle around $p^{\prime}$. Call a point even if it has an even number of points before it in this ordering. Call a point odd if it has an odd number of points before it in this ordering. If $e$ is incident to an odd point, then we embed the parent of $u$ into this point. The remaining subtree rooted at $u$ can be embedded without using $e$. If the endpoints of $e$ are both even, between them there is an odd point. We embed the parent of $u$ into this point. The remaining vertices can be embedded without using $e$ (see Figure 47.

The upper bound of $f(n, n) \leq 3$ of Theorem 1.2 follows directly from Lemma 3.2 . Now we prove in Lemma 2.2 and Theorem 2.3, that if $G$ is a convex geometric graph, at least three edges are needed to forbid some tree on $n$ vertices.

Lemma 2.2 Let $T$ be a tree on $n$ vertices. If $G$ is a convex geometric graph, then $T$ can be embedded into $G$ using less than $\frac{n}{2}$ convex hull edges of $G$.

Proof: If $T$ is a star, then any embedding of $T$ into $G$ uses only two convex hull edges. If $T$ is a path then it can be embedded into $G$ using at most two convex hull edges. Therefore, we may assume that $T$ is neither a star nor a path.

Since $T$ is not a path, it has a vertex of degree at least three. Choose this vertex as the root. Since $T$ is not a star, the root has a child whose subtree has at least two nodes. Order the children of $T$ so that this node is first. Embed $T$ into $G$ with the embedding algorithm.

Let $u$ and $v$ be vertices of $T$, so that $u$ is the parent of $v$. Suppose that the subtree rooted at $v$ has at least two nodes. Then in the embedding algorithm we have at least two choices to embed $v$ once the ordering 

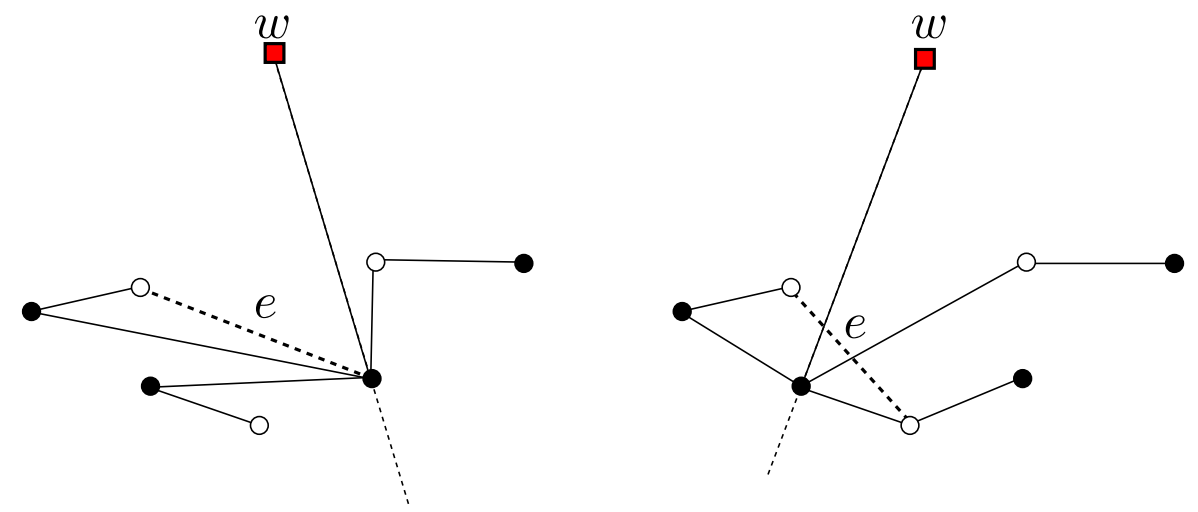

Fig. 4: The two sub-cases, when $u$ has a grandparent $w$, and all the subtrees of its children are edges. Odd points are painted in black and even points in white. The forbidden edges are dashed.

of the children of $u$ has been chosen. At least one of the choices is such that $u v$ is not embedded into a convex hull edge. Therefore, we may assume that the embedding is such that each convex hull edge used, is incident to a leaf.

Note that every vertex of $T$, distinct from the root, is incident to at most one convex hull edge in the embedding. Since the first child of the root is not a leaf, no convex hull edge is used to embed this child. Only in the embedding of the last child of the root a convex hull may have been used. Therefore every vertex of $T$ is incident to at most one convex hull edge. Thus the set of convex hull edges used in the embedding is a matching. Therefore at most $n / 2$ convex hull edges are used in the embedding.

Suppose that exactly $n / 2$ convex hull edges are used. One of these edges must be incident to the root. Since the root was chosen of degree at least three it has a child which is not a leaf nor the first child; we place this vertex last in the ordering of the children of the root. The leaf adjacent to the root can no longer be a convex hull edge and the embedding uses less than $n / 2$ convex hull edges.

Theorem 2.3 If $G$ is a convex geometric graph and has at most two forbidden edges, then any tree on $n$ vertices can be embedded into $G$, without using a forbidden edge.

Proof: Let $f_{0}$ be an embedding given by Lemma 2.2, of $T$ into $G$. For $0 \leq i \leq n$, let $f_{i}$ be the embedding produced by rotating $f_{0}, i$ places to the right. Assume that in each of these rotations at least one forbidden edge is used, as otherwise we are done. Let $e_{1}, \ldots, e_{m}$ be the edges of $T$ that are mapped to a forbidden edge in some rotation. Assume that the two forbidden edges are an $l$-edge and an $r$-edge respectively.

Suppose that $l \neq r$. Then, each edge of $T$ can be embedded into a forbidden edge at most once in all of the $n$ rotations. Thus $m \geq n$. This is a contradiction, since $T$ has $n-1$ edges.

Suppose that $l=r$. Then, each of the $e_{i}$ is mapped twice to a forbidden edge. Thus $m \geq n / 2$. By Lemma 2.2, $f_{0}$ uses less than $n / 2$ convex hull edges. Therefore, $l=r>0$. But a set of $n / 2$ or more $r$-edges, with $r>0$, must contain a pair of edges that cross. And we are done, since $f_{0}$ is an embedding. 


\section{Bounds on $f(n, k)$}

In this section we prove Theorem 1.1. First we show the upper bound.

Lemma 3.1 If $T_{n}$ is embedded into $G_{n}$ then every edge incident to a leaf of $T_{n}$ must be embedded into a convex hull edge.

Proof: Let $e:=u v$ be an edge of $T_{n}$ incident to leaf. Suppose that $u$ is the leaf vertex. Then $v$ is of degree two. Suppose that $e$ is not embedded into a convex hull edge of $G$. Then $e$ divides $S \backslash\{u, v\}$ into two non-empty subsets $S_{1}$ and $S_{2}$, so that $S_{1}$ lies on the opposite side of $S_{2}$ with respect to $e$. Assume that the parent of $v$ is embedded into $S_{1}$. Then no vertex of $T_{n}$ can be embedded into $S_{2}$ without crossing $e$. Therefore $e$ must be a convex hull edge of $G$.

Lemma 3.2 If $G$ is a convex geometric graph, then forbidding three consecutive convex hull edges of $G$ forbids the embedding of $T_{n}$.

Proof: Recall that $T_{n}$ comes from subdividing a star, let $v$ be the non leaf vertex of this star. Let $p_{1} p_{2}, p_{2} p_{3}, p_{3} p_{4}$ be the forbidden edges, in clockwise order around the convex hull of $G$. Note that by Lemma 3.1, in any embedding of $T_{n}$ into $G$, an edge incident to a leaf of $T_{n}$, must be embedded into a convex hull edge. Neither the leaves of $T_{n}$ nor its neighbors can be embedded into $p_{2}$ or $p_{3}$, without using a forbidden edge. Thus, $v$ must be embedded into $p_{2}$ or $p_{3}$. Without loss of generality assume that $v$ is embedded into $p_{2}$. But then, the embedding must use $p_{2} p_{3}$ or $p_{3} p_{4}$

Lemma 3.3 If $G$ is a convex geometric graph, then forbidding the convex hull edges incident to any three vertices $p_{1}, p_{2}$ and $p_{3}$ of $G$, forbids the embedding of $T_{n}$.

Proof: Note that by Lemma 3.1 neither a leaf of $T_{n}$, nor its neighbor can be embedded into $p_{1}, p_{2}$ or $p_{3}$, without using a forbidden edge. But at most two points do not fall into this category.

$$
\text { Lemma 3.4 } \quad f(n, k) \leq 2 \frac{n(n-2)}{k-2}
$$

Proof: Let $G$ be a complete convex geometric graph. We forbid every $r$-edge of $G$ for $r=0, \ldots$, $\left\lceil 2 \frac{n-2}{k-2}-2\right\rceil$. Note that, in total we are forbidding at most $n\left(\left\lceil 2 \frac{n-2}{k-2}-2\right\rceil+1\right) \leq 2 \frac{n(n-2)}{k-2}$ edges. As every subset of points of $G$ is in convex position, it suffices to show that every induced subgraph $H$ of $G$ on $k$ vertices is in one of the two configurations of Lemmas 3.2 and 3.3

Assume then, that $H$ does not contain three consecutive forbidden edges in its convex hull nor three vertices, each with its two convex hull edges forbidden. $H$ has at most two (non-adjacent) pairs of consecutive forbidden edges in its convex hull. Therefore every forbidden edge of $H$ in its convex hull—with the exception of at most two-must be preceded by an $\ell$-edge (of $G$ ), with $\ell>\left\lceil 2 \frac{n-2}{k-2}-2\right\rceil$. The number of these $\ell$-edges contained in $H$ is at least $\frac{k-2}{2}$. The points separated by these edges amount to more than $\frac{k-2}{2}\left[2 \frac{n-2}{k-2}-2\right] \geq n-k$ points of $G$. This is a contradiction, since together with the $k$ points of $H$ this is strictly more than $n$. 
Now, we show the lower bound of Theorem 1.1 .

$$
\text { Lemma } 3.5 \quad f(n, k) \geq\left(\frac{1}{2}\right) \frac{n^{2}}{k-1}-\frac{n}{2}
$$

Proof: Let $F$ be a set of edges whose removal from $G$ forbids some $k$-tree. Let $H:=G \backslash F$. Note that $H$ contains no complete $K_{k}$ as a subgraph, otherwise any $k$-tree can be embedded into this subgraph. By Turán's Theorem [7], $H$ cannot contain more than $\left(\frac{k-2}{k-1}\right) \frac{n^{2}}{2}$ edges. Thus $F$ must have size at least

$$
\left(\begin{array}{l}
n \\
2
\end{array}\right)-\left(\frac{k-2}{k-1}\right) \frac{n^{2}}{2}=\left(\frac{1}{2}\right) \frac{n^{2}}{k-1}-\frac{n}{2}
$$

\section{Acknowledgments}

Part of this work was done at the "First Workshop in Combinatorial Optimization at Cinvestav". It was continued during a visit of L. Barba, R. Fabila-Monroy, J. Leaños and G. Salazar to Abacus research center(ii)

\section{References}

[1] O. Aichholzer, S. Cabello, R. Fabila-Monroy, D. Flores-Peñaloza, T. Hackl, C. Huemer, F. Hurtado, and D. R. Wood. Edge-removal and non-crossing configurations in geometric graphs. Discrete Math. Theor. Comput. Sci., 12(1):75-86, 2010.

[2] L. F. Barba, R. Fabila-Monroy, D. Lara, J. Leaños, C. Rodríguez, G. Salazar, and F. Zaragoza. The Erdős-Sós conjecture for geometric graphs. In Proc. 28th European Worskhops in Computational Geometry, EUROCG '12, Asissi, Italy, 2012.

[3] P. Bose, M. McAllister, and J. Snoeyink. Optimal algorithms to embed trees in a point set. J. Graph Algorithms Appl., 1(2):15 pp. (electronic), 1997.

[4] A. García, C. Hernando, F. Hurtado, M. Noy, and J. Tejel. Packing trees into planar graphs. J. Graph Theory, 40(3):172-181, 2002.

[5] Y. Ikebe, M. A. Perles, A. Tamura, and S. Tokunaga. The rooted tree embedding problem into points in the plane. Discrete Comput. Geom., 11(1):51-63, 1994.

[6] G. Károlyi, J. Pach, G. Tóth, and P. Valtr. Ramsey-type results for geometric graphs. II. Discrete Comput. Geom., 20(3):375-388, 1998.

[7] P. Turán. On an extremal problem in graph theory. Mat. Fiz. Lapok, 48:436-452, 1941.

(ii) ABACUS, CONACyT grant EDOMEX-2011-C01-165873 